\section{Stellenwert chirurgischer Operationsverfahren
bei der Diagnostik und
Therapie der Tuberkulose Operationsverfahren
bei der Diagnostik und
Therapie der Tuberkulose Operationsverfahren
bei der Diagnostik und
Therapie der Tuberkulose}

Zusammenfassung: In den letzten Jahrzehnten hat die Anzahl der chirurgischen Eingriffe bei der Behandlung der Lungentuberkulose stetig abgenommen. Trotz effektiver antituberkulotischer Medikamente bestehen aber weiterhin Indikationen zur chirurgischen Intervention. Diese Studie wurde unternommen, um den aktuellen Stellenwert der Chirurgie bei Diagnostik und Therapie der Tuberkulose zu bestimmen. Aus diesem Grund wurden alle operierten Patienten mit Lungentuberkulose zwischen 1988 und 1999 analysiert. In 2\% aller Patienten mit Lungentuberkulose wurde eine chirurgische Indikation gestellt $(n=35)$. Dabei waren Resektionen zum Ausschluss eines Malignoms die häufigste Operationsindikation, gefolgt von multiresistenter Tuberkulose und eingeschränkter Patienten-Compliance bei der Medikamenteneinnahme, Dekortikation bei Kavernenruptur und spezifischem Empyem, Hämoptoe und „destroyed lung“. Eine weitere chirurgische Indikation war die Behandlung von Aspergillomen in postspezifischen Hohlräumen. Postoperative Komplikationen wie erhöhter Blutverlust $(>1000 \mathrm{ml}$ ) oder einer broncho-pleuralen Fistel traten in $37 \%$ der Fälle auf, keiner der Patienten verstarb. Chirurgische Eingriffe stellen weiterhin eine zusätzliche Option bei der Behandlung der Lungentuberkulose dar. Einer Operation muß eine ausreichende antituberkulotische Chemotherapie vorausgehen. Aufgrund einer erhöhten Morbidität sollte die Entscheidung zur chirurgischen Intervention individuell vom Allgemeinzustand des Patienten abhängig gemacht werden. In $10 \%$ aller operierten Patienten mit unklarem Rundherd $(n=1132)$ war das Infiltrat spezifischer Ätiologie.

The Role of Thoracic Surgery in Diagnosis and Therapy of Pulmonary Tuberculosis: Over the last decades the annual number of surgical intervention for treatment of pulmonary tuberculosis has steadily declined. Despite effective antituberculotic medication, there are still indications for thoracic surgery. The aim of the present study was to give an account of the diagnostic and therapeutic role of surgery of pulmonary tuberculosis. Therefore we analysed all operated patients with pulmonary tuberculosis between 1988 and 1999. In $2 \%$ of all patients with pulmonary tuberculosis surgical intervention was performed $(n=35)$. Their main indication was resection for suspected carcinoma, multi-drug resistance or non-compliance to the medical treatment, decortication because of cavern rupture or empyema, hemoptoe and destroyed lung. A additional indi-

Pneumologie 2000; 54: $318-323$

(C) Georg Thieme Verlag Stuttgart · New York ISSN 0934-8387
H. Geldmacher, C. Kroeger, D. Branscheid, J. Schatz, H. Magnussen, D. Kirsten

Krankenhaus Großhansdorf, Zentrum für Pneumologie und Thoraxchirurgie, Großhansdorf cation for surgery was pulmonary aspergilloma caused by superinfection of postspecific cavities. Postoperative complications like bleeding (> $1000 \mathrm{ml}$ ) or broncho-pleural fistula occurred in $37 \%$, none of the patients died. Surgery is still a valid option für the treatment of pulmonary tuberculosis. Before surgical intervention, a long term antituberculotic chemotherapy is essential. Patients have to be selected individually because of an increased morbidity. In $10 \%$ of all operated patients $(n=1132)$ with preoperative unidentifiable pulmonary mass the infiltrate was specific aetiology.

\section{Einleitung}

Seit Einführung der antibiotischen Chemotherapie bei der Behandlung der Tuberkulose ist die Anzahl chirurgischer Eingriffe deutlich zurückgegangen [1]. Bildeten früher kollapschirurgische und lungenresezierende Verfahren zur Behandlung und Rezidivprophylaxe der Tuberkulose einen hohen prozentualen Anteil an thoraxchirurgischen Eingriffen, werden in den westlichen Industrienationen heute Operationsindikationen nur noch selten gestellt. Mitte der achtziger bezifferten zahlreiche klinische Studien den prozentualen Anteil operierter Tuberkulose-Patienten noch zwischen 5 und $15 \%[2,3]$. Bedingt durch die weiterhin rückläufige Inzidenz der Tuberkulose in Deutschland liegt der aktuelle Anteil der chirurgisch versorgten Patienten mit spezifischen Lungenerkrankungen bei unter einem Prozent [4]. Trotz effektivem Einsatz antituberkulotischer Medikamente bestehen in Deutschland jedoch weiterhin akute und elektive Indikationen zur chirurgischen Intervention bei gesicherter und medikamentös behandelter Tuberkulose. In Ländern mit steigender Erregerresistenz bei gleichzeitig unzureichender medikamentöser Versorgung erleben lungenresezierende Verfahren sogar eine Renaissance [5].

Eine andere Indikationsgruppe zur chirurgischen Intervention bei spezifischen Läsionen stellen die Patienten mit einer malignomverdächtigen pulmonalen Raumforderung dar, die unter Tumorverdacht operiert werden und deren Lungenveränderung sich makroskopisch und histologisch als spezifischer Genese erweist. Hierzu zählen auch Patienten mit radiologischen Veränderungen in postspezifischen Lungenherden, die fälschlicherweise als Narbenkarzinom interpretiert und chirurgisch entfernt werden.

Vor dem Hintergrund erhöhter postoperativer Mortalität und Morbidität bei Resektionen spezifischer Herde [6,7] besteht 
weiterhin Interesse an einer klinischen Analyse, um den aktuellen Stellenwert thoraxchirurgischer Eingriffe bei der Therapie der Tuberkulose zu definieren.

\section{Methodik}

Die Krankenakten aller Patienten, die zwischen 1988 und 1999 wegen eines tumorsuspekten Rundherdes $(n=112)$ im Krankenhaus Großhansdorf operiert worden sind, wurden retrospektiv analysiert und der Anteil spezifischer Lungenveränderungen bestimmt.

Als zweite Patientengruppe wurden alle im gleichen Zeitraum operierten Patienten mit gesicherter Tuberkulose $(n=35)$ analysiert. Bei beiden Indikationsgruppen wurden separat der prä- und postoperative Verlauf ausgewertet und mit älteren Analysen und Publikationen aus unserer Klinik verglichen.

Zum Patientenkollektiv zählten Patienten aus dem Bereich Hamburg, Schleswig-Holstein und dem nördlichen Niedersachsen.

\section{Ergebnisse}

\section{Diagnostische Thorakotomie bei Patienten mit unklarem Rundherd}

In den Jahren zwischen 1988 und 1999 wurden im Krankenhaus Großhansdorf 112 Patienten wegen einer präoperativ unklaren pulmonalen Raumforderung operiert, deren histologische Aufarbeitung des Resektates eine Tuberkulose als Ursache der Lungenveränderung ergab. Dieses entspricht einem prozentualen Anteil von 9,9\% aller Patienten, die während dieser Zeit wegen eines Malignoms suspekten Raumforderung diagnostisch thorakotomiert worden sind $(\mathrm{n}=1132)$. Prädisponierende Faktoren (Alkoholismus, Diabetes mellitus, frühere Mykobakterien-Infektion, generelle Immunsuppression etc.) waren nur bei einer Minorität beschrieben (15\% der Patienten), die Hälfte der Patienten waren Raucher. Das Patientenkollektiv unterschied sich weder in der Altersstruktur noch Nationalität von der Gesamtpatientenpopulation (Tab. 1).

Topographisch lagen über 52\% der pulmonalen Raumforderungen im Bereich des rechten oder linken Lungenoberfeldes, gefolgt von den Mittelfeldern (30\%) und den Lungenunterfeldern (8\%). In ca. 6\% der Fälle ließen sich beidseitige Herde radiologisch nachweisen.

Eine präoperative Tuberkulosediagnostik (Tuberkulin-Test, Mykobakterien-Kulturen aus Bronchialsekret oder Biopsie) wurde nur in $47,5 \%$ der Patienten durchgeführt, bei den restlichen Patienten führte die Diagnostik zu keinem definitiven Nachweis von Tuberkuloseerregern. Trotz negativer Tuberkulosekulturen wurde bei 21 Patienten präoperativ eine antituberkulotische Verdachtstherapie ambulant eingeleitet (Tab. 2), wegen mangelnder radiologischer Infiltratrückbildung wurde jedoch die Indikation zur diagnostischen Thorakotomie gestellt.

Bei nahezu 90\% der Patienten wurden die spezifischen Lungenherde mittels Keilresektionen entfernt, in 10\% waren Segment-, bzw. Lappenresektionen nötig. Der mikrobiologi-
Tab. 1 Demographische Merkmale 112 operierter Patienten mit spezifischem Rundherd

\begin{tabular}{|c|c|c|}
\hline & Patientenzahl & $\begin{array}{l}\text { prozentualer } \\
\text { Anteil (\%) }\end{array}$ \\
\hline \multicolumn{3}{|l|}{ Geschlecht } \\
\hline weiblich & 46 & 41,0 \\
\hline männlich & 66 & 59,0 \\
\hline \multicolumn{3}{|l|}{ Nationalität } \\
\hline Deutschland & 102 & 91,1 \\
\hline Ausland & 10 & 9,9 \\
\hline \multicolumn{3}{|l|}{ Immunsuppression } \\
\hline früherer Mykobakterieninfekt & 10 & 8,9 \\
\hline Alkoholismus & 5 & 4,4 \\
\hline Diabetes mellitus & 4 & 3,6 \\
\hline HIV & 1 & 0,8 \\
\hline immunsupp. Therapie & 1 & 0,8 \\
\hline keine & 91 & 81,5 \\
\hline \multicolumn{3}{|l|}{ Röntgenherde im Röntgenbild } \\
\hline Rundherd im OF links & 23 & 20,5 \\
\hline Rundherd im OF rechts & 36 & 32,1 \\
\hline Rundherd im MF links & 18 & 16,1 \\
\hline Rundherd im MF rechts & 16 & 14,3 \\
\hline Rundherd im UF links & 5 & 4,5 \\
\hline Rundherd im UF rechts & 4 & 3,6 \\
\hline Rundherde beidseits & 10 & 9,0 \\
\hline \multicolumn{3}{|l|}{$\begin{array}{l}\text { präoperative antituberkulotische } \\
\text { Therapie }\end{array}$} \\
\hline ja & 21 & 18,8 \\
\hline nein & 91 & 81,2 \\
\hline
\end{tabular}

sche Erregernachweis aus sezerniertem Gewebe gelang in 31 Fällen $(28,4 \%)$, eine medikamentöse Tuberkulosetherapie wurde in 71 Fällen $(63,4 \%)$ eingeleitet. Intra- und postoperative Komplikationen wie verstärkte Blutungen traten nur in unter $4 \%$ auf.

\section{Chirurgische Eingriffe bei Patienten mit präoperativ gesicherter Tuberkulose}

Von 1750 Patienten, die in den Jahren von 1988 bis 1999 im Krankenhaus Großhansdorf wegen einer Tuberkulose behandelt worden sind, wurde in 35 Fällen (2\%) trotz antituberkulotischer Therapie eine Operationsindikation gestellt. Die demographischen und klinischen Merkmale zeigt Tab. $\mathbf{3}$

$\mathrm{Zu}$ den häufigsten Indikationen zählten Thorakotomien bei therapieresistentem Lungeninfiltrat zum Ausschluss einer zusätzlichen malignen Grunderkrankung (in 23\% der Fälle). Bei $20 \%$ der Patienten wurde eine Lungenresektion bei multiresistenter Tuberkulose (MDR) durchgeführt, dabei bestand bei einem Patienten gleichzeitig eine stark eingeschränkte Patienten-Compliance. In weiteren $17 \%$ wurde eine Indikation zur Pleurektomie bei Kavernenruptur gestellt. Weitere Indikationen zur Lungenresektion waren superinfizierte Kavernen 
Tab. 2 Präoperative Diagnostik, Operationsverfahren und Therapiekonsequenz der 112 operierten Patienten mit spezifischem Rundherd

\begin{tabular}{lcl}
\hline & Patientenzahl & $\begin{array}{c}\text { prozentualer } \\
\text { Anteil (\%) }\end{array}$ \\
\hline Tuberkulintest & & \\
positiv & 53 & 47,4 \\
negativ & 8 & 7,1 \\
nicht bestimmt & 51 & 45,5 \\
präoperative Diagnostik (Bronchoskopie/transthorakale Biopsie) \\
ohne Tbb-Kulturen & 59 & 52,6 \\
mit Tbb-Kulturen & 53 & 47,4 \\
Operationsverfahren & & \\
Keilresektion & 85 & 75,9 \\
Mehrfachkeilresektionen & 15 & 13,3 \\
Segmentresektion & 4 & 3,6 \\
Lappenresektion & 8 & 7,2 \\
zusätzl. Pleurektomie & 14 & 12,5 \\
mikrobiologischer Erregernachweis & \\
bestimmt & 109 & 97,3 \\
$\quad$ positiv & 31 & 28,4 \\
negativ & 78 & 71,6 \\
nicht bestimmt & 31 & 2,7 \\
postoperative medikamentöse Behandlung & \\
ja & 71 & 63,4 \\
$\quad$ mit Erregernachweis & 31 & \\
nein & 40,3 \\
\hline
\end{tabular}

Tab. 3 Demographische und klinische Merkmale der 35 Patienten mit gesicherter Tuberkulose

\begin{tabular}{lll}
\hline & Patientenzahl & $\begin{array}{l}\text { prozentualer } \\
\text { Anteil (\%) }\end{array}$ \\
\hline $\begin{array}{l}\text { Geschlecht } \\
\text { weiblich } \\
\text { männlich }\end{array}$ & 14 & 40,0 \\
Nationalität & 21 & 60,0 \\
$\begin{array}{l}\text { Deutschland } \\
\text { Ausland }\end{array}$ & 26 & 74,3 \\
Immunsuppression & 9 & 25,7 \\
Alkoholismus & & \\
früherer Mykobakterieninfekt & 8 & 11,4 \\
Bronchialkarzinom & 4 & 22,8 \\
Diabetes mellitus & 2 & 5,7 \\
HIV & 2 & 5,7 \\
keine & 1 & 2,9 \\
\hline
\end{tabular}

(Aspergillom) in 14\% der Fälle, Dekortikationen und Pleuraausräumungen bei tuberkulöser Pleuritis (11\%), massive Hämoptoe im spezifisch veränderten Lungengewebe (9\%) und in 6\% die Entfernung spezifisch zerstörter Lungenlappen (destroyed lung).

Wie in der Tab. 4 sichtbar, wurden bei diagnostischen Thorakotomien bei malignom-suspekten Herden überwiegend Keiloder Segmentresektionen gewählt mit vergleichsweise geringem Blutverlust und seltenen postoperativen Komplikationen. Lappenresektionen waren bei Aspergillomen das Operationsverfahren der Wahl, ähnlich wie bei der Infektsanierung multi-resistenter Tuberkulose. Bei akuter Lungenblutung war nur bei ${ }^{1} / 3$ der Fälle eine Lobektomie möglich, bei den übrigen Patienten musste eine Pneumonektomie erfolgen. Auch bei Patienten mit destroyed lung ließ sich keine anatomische Teilresektion durchführen. Pleuraoperationen bei tuberkulöser Pleuritis oder Kavernenruptur zeigten eine deutlich stärkere Blutungsneigung und damit einen größeren intraoperativen Blutverlust. Auch traten gleichzeitig vermehrt postoperative Komplikationen wie bronchopleurale Fisteln, verstärkte Nachblutungen, spezifische oder unspezifische Empyeme auf.

Wie in Abb.1 sichtbar, ist bis Anfang 1990 der prozentuale Anteil operierter Patienten mit spezifischen Lungenerkrankungen stetig abnehmend. So sank der prozentuale Anteil tuberkulöser Lungeneingriffe von über 35\% (1965) auf 0,9\% (1992). Auffällig ist jedoch eine leichtgradige Zunahme der chirurgischen Eingriffe seit 1992 auf 3,2\% 1998.

\section{Diskussion}

In der vor-chemotherapeutischen Ära der Tuberkulose stellte die chirurgische Therapie die einzige Behandlungsmöglichkeit der Lungentuberkulose dar. Bei einer Letalität der unbehandelten Tuberkulose von 50\% konnte mit der Kollapstherapie in bis $\mathrm{zu} 70 \%$ der Fälle kavernöser Lungentuberkulose dauerhaft kuriert werden [8,9]. Zahlen der 40er und 50er Jahre bezifferten die Rezidivfreiheit nach Operationen auf über 90\% [10]. So zeigen Daten aus unserer Klinik, dass 1965 über 35\% aller Thoraxoperationen (ca. 400) zur Behandlung spezifischer Lungenerkrankungen vorgenommen wurden [11]. Die kollapschirurgischen Eingriffe führten zu einer Reihe von Frühkomplikationen, wie die Entwicklung spezifischer Empyeme oder broncho-pleuraler Fisteln mit Mischinfektionen. Als Spätfolgen traten nahezu regelmäßig restriktive Ventilationsstörungen mit Cor pulmonale auf [12]. Nach Einführung der antituberkulotischen Chemotherapie war die Anzahl der spezifischen Lungenoperationen rückläufig bedingt durch Ausheilung und Rezidivfreiheit von über 95\% [13].

Versucht man die Häufigkeit der Operationen wegen Tuberkulose zu bestimmen, vermischt sich diese Gruppe mit anderen Indikationen. So werden oftmals Patienten in den Operationsstatistiken aufgeführt, die primär nicht wegen einer Tuberkulose, sondern wegen einer tumorsuspekten Raumforderung zum Ausschluss eines Malignoms thorakotomiert wurden. Diese Gruppe wird deshalb gesondert diskutiert. 
Tab. 4 Indikationen, Operationsverfahren und postoperative Komplikationen bei 35 Patienten mit gesicherter Tuberkulose

\begin{tabular}{|c|c|c|c|c|c|c|c|}
\hline & $\begin{array}{l}\text { therapie- } \\
\text { resistentes Infiltrat }\end{array}$ & $\begin{array}{l}\text { Multi-Drug- } \\
\text { Resistance* }\end{array}$ & $\begin{array}{l}\text { Kavernenruptur } \\
+ \text { spez. Empyem }\end{array}$ & Aspergillom & Pleuraschwiele & Hämoptysen & $\begin{array}{l}\text { Destroyed } \\
\text { lung }\end{array}$ \\
\hline Gesamtzahl & 8 & 7 & 6 & 5 & 4 & 3 & 2 \\
\hline \multicolumn{8}{|l|}{ Operationsverfahren } \\
\hline Pneumonektomie & & & & & & 2 & 2 \\
\hline Lappenresektion & 1 & 7 & $2^{* *}$ & 5 & & 1 & \\
\hline Segmentresektion & 1 & & & & & & \\
\hline Keilresektion & 6 & & $4^{* *}$ & & & & \\
\hline Ausräumung des Pleuraraumes & & & 2 & & & & \\
\hline Dekortikation & & & & & 4 & & \\
\hline \multicolumn{8}{|l|}{ Blutverlust } \\
\hline$<500 \mathrm{ml}$ & 8 & 3 & 6 & 2 & & 1 & \\
\hline $500-1000 \mathrm{ml}$ & & 4 & & 5 & 2 & & \\
\hline$>1000 \mathrm{ml}$ & & & & & 2 & 2 & 2 \\
\hline \multicolumn{8}{|l|}{ Komplikationen } \\
\hline Drainagezeit > 7 Tage & & 3 & 1 & 1 & 1 & & \\
\hline unspez. Empyem & & & 1 & & & & \\
\hline
\end{tabular}

* Bei zwei Patienten bestand zusätzlich eine eingeschränkte Compliance bei der Medikamenteneinnahme

** Bei diesen Operationen wurde gleichzeitig eine Pleurektomie durchgeführt

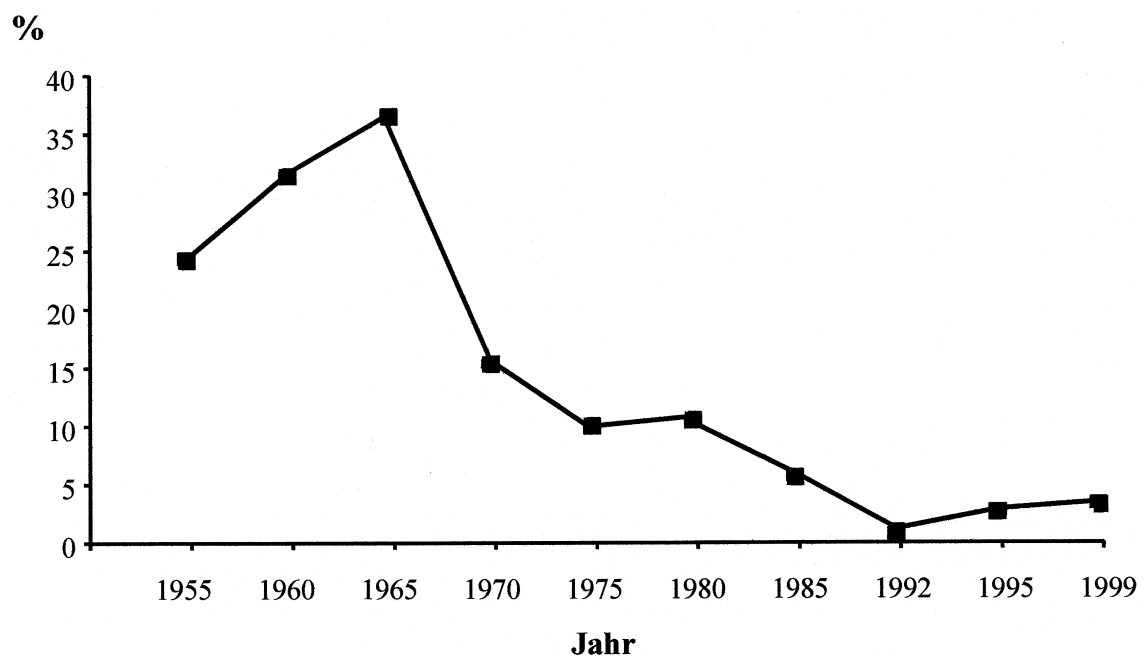

Abb. 1 Anteil operierter spezifischer Lungenerkrankungen im Verhältnis zur Gesamtzahl thoraxchirurgischer Eingriffe in Großhansdorf.

In unserem Patientenkollektiv war in ca. $76 \%$ der Patienten die diagnostische Thorakotomie zum Ausschluss eines Bronchialkarzinoms die häufigste Operationsindikation der spezifischen Lungenerkrankungen. Dieses entspricht einem prozentualen Anteil von 10\% aller diagnostischen Thorakotomien, die seit 1988 bei unklaren Rundherden durchgeführt worden sind. Verglichen mit in der Schweiz publizierten Daten von Lardinois et al. liegt damit der prozentuale Anteil diagnostischer Eingriffe deutlich höher [14].

Wie auch bei anderen spezifischen Erkrankungen waren nur bei einem geringen Anteil der analysierten Patienten prädisponierende Faktoren nachweisbar [15]. Dagegen zeigten die demographischen Patientendaten keinerlei Unterschiede im
Alter, Geschlechtsverhältnis oder Herkunftsland, verglichen mit dem onkologischen Patientenkollektiv. Ein gemeinsames Vorkommen maligner und spezifischer Erkrankungen ist bekannt [16].

In über $75 \%$ der Fälle ließ sich der suspekte Lungenherd mittels Keilresektion ohne erhöhte postoperative Komplikationsrate entfernen. Eine anschließende medikamentöse Behandlungskonsequenz ergab sich in $63 \%$ der Fälle, obwohl ein positiver mikrobiologischer Erregernachweis nur in $43 \%$ dieser Patientengruppe gelang. Diese Ergebnisse stehen in Übereinstimmung mit publizierten Sensitivitätsbestimmungen für mikrobiologische Nachweisverfahren aus Gewebe [17], bei denen die Sensitivität zwischen 40 und 60\% angege- 
ben wurde. Bei den restlichen 57\% wurde die Indikation zur antituberkulotischen Anschlussbehandlung aufgrund des histologischen Nachweises einer aktiven verkäsenden Epitheloidzellgranulomatose mit Umgebungsherden gestellt.

Die häufigste Operationsindikation bei Patienten mit gesicherter Lungentuberkulose war eine diagnostische Thorakotomie bei verzögerter bzw. fehlender Infiltratrückbildung trotz antituberkulotischer Therapie. Diese Indikation nimmt eine Zwischenrolle ein, da sie weder der Gruppe der unklaren Rundherde, noch der Gruppe der chirurgischen Therapieindikationen bei gesicherter Tuberkulose zuzuordnen ist.

Bei 20\% der Patienten wurde eine Indikation zur Lungenresektion wegen eingeschränkter Medikamentensensibilität (Multiresistenz) gestellt. Für diese Operation eignen sich vornehmlich Patienten mit umschriebenen, nicht disseminierten Infektionsarealen, die unter medikamentöser Vielfach-Therapie keine Tendenz zur Regression zeigen $[4,18]$. Bei zwei Patienten bestand zusätzlich eine „soziale Indikation“ [19] (eingeschränkte Compliance bei unsicherer Medikamenteneinnahme; Immunsuppression bei HIV-Infektion). In der Regel sollte dem Eingriff eine mehrmonatige antituberkulotische Behandlung vorausgehen, um nach Möglichkeit eine Sputumkonversion bzw. eine deutliche Reduktion der Erregermenge präoperativ zu erzielen [20,21]. Mit einer postoperativen Fortführung der antituberkulotischen Therapie ist bei der Mehrheit der operierten Patienten eine kulturelle Sputumkonversion erreicht worden. In Übereinstimmung mit anderen Publikationen aus westlichen Industrienationen (Spanien, Frankreich, USA) stellt die chirurgische Intervention bei multiresistenten Tuberkuloseerregern mittlerweile die häufigste Operationsindikation bei Lungentuberkulose dar $[18,22,23]$. So ist auch zu erklären, dass in unserer Analyse nach 1992 wieder eine leichte Zunahme der Operationen zu registrieren ist.

Eine weitere Indikation zur chirurgischen Therapie bilden spezifische Erkrankungen des Rippenfells. In unserer Studie nahmen die Kavernenrupturen mit Pneumothorax, die konservativ nicht behandelt werden konnten, die größte Gruppe ein. Hier wurde mittels Keilresektion die broncho-pleurale Fistel übernäht, gleichzeitig führte eine Pleurektomie zu einem Verkleben der Pleurablätter. Dabei reichte in zwei Fällen zur Deckung der Fistel eine Keilresektion nicht aus, so dass ein Lungenlappen reseziert werden musste. Bei weiteren zwei Patienten führte die Ruptur der kavernösen Lungenveränderungen zu einem spezifischen Empyem. Um erheblichen restriktiven Ventilationsstörungen bei tuberkulöser Pleuritis entgegenzuwirken, wurde bei 4 Patienten eine Indikation zur Dekortikation und Entfernung der Pleuraverschwartungen gestellt. Auffällig dabei waren hierbei ein deutlich erhöhter intraoperativer Blutverlust $(>1000 \mathrm{ml}$ ) und postoperativ verlängerte Drainagezeiten bedingt durch broncho-pleurale Fisteln (> 7 Tage).

Gelegentlich führen tuberkulöse Folgeerkrankungen zu akuten Operationsindikationen. Dabei führen sekundäre Pilzinfektionen tuberkulöser Hohlräume zu Erosionen der Bronchialschleimhaut, die massive, operativ zu behandelnde Hämoptysen verursachen können. Dauerhafte Erfolge durch systemische oder lokal verabreichte antimykotische Medikamente zur Reduktion der Blutungskomplikation werden kon- trovers diskutiert [24-27]. Eine massive Hämoptoe ( $>500 \mathrm{ml}$ in 24 Stunden) stellt in jedem Fall eine absolute Operationsindikation dar [3]. Derartige Notoperationen weisen eine erhöhte Operationsletalität auf $[28,29]$. Daher sehen einige Autoren auch in einem asymptomatischen Aspergillom bereits eine Operationsindikation $[4,28,30]$.

Selten werden spezifisch destruierte Lungenlappen zur Vermeidung von Sekundärkomplikationen wie Bronchiektasen, Hämoptoe, sekundärer Pilzinfektion oder Reaktivierung präventiv lungenreseziert [7]. Die zerstörten Lungenanteile (destroyed lobe/-lung) werden als Endzustand einer ausgeprägten Lungentuberkulose infolge chronisch-entzündlicher Verläufe beobachtet [31]. In unserer Studie wurde bei zwei Patienten eine Operationsindikation gestellt, jedoch war eine anatomische, lappenbegrenzende Resektion technisch nicht möglich, so dass eine Pneumonektomie erforderlich wurde. Aufgrund entzündlicher Verwachsungen zeigte sich intraoperativ ein massiver Blutverlust (in einem Fall bis zu 4,5 l). Mit dem Wissen einer erhöhten postoperativen Letalität durch intraoperativ gesteigerte Blutungsneigung $[5,7,32]$ und dem postoperativen Risiko einer broncho-pulmonalen Fistel sollte vergleichbar mit der Aspergillomresektion ein chirurgisches Vorgehen streng vom Gesamtzustand des Patienten abhängig gemacht werden.

\section{Schlussfolgerung}

Von allen thoraxchirurgischen Interventionen, bedingt durch eine Tuberkulose, bilden diagnostische Thorakotomien zum Ausschluss eines Bronchialkarzinoms mit ca. ${ }^{3} / 4$ aller Eingriffe den größten Anteil. Es ist zu hoffen, dass durch verbesserte bildgebende Verfahren die Differentialdiagnostik so verbessert werden kann, dass die Anzahl solcher Eingriffe deutlich verringert wird. Trotz effektiver medikamentöser Behandlungsmöglichkeiten der Tuberkulose bestehen weiterhin Indikationen zur thoraxchirurgischen Intervention. Dabei nehmen Lungenresektionen bei multiresistentem Erreger eine wichtige, aber derzeit nur selten erforderliche Rolle ein. Weitere Indikationen betreffen spezifische Erkrankungen des Pleuraraumes. Bei allen derartigen Operationen ist aufgrund der entzündlichen Lungen- und Pleuraveränderungen mit erhöhtem intraoperativen Blutverlust und postoperativen Komplikationen (z.B. broncho-pleuraler Fistel) zu rechnen. Den Operationen sollte eine mehrmonatige Chemotherapie vorausgehen; auch postoperativ muss die antituberkulotische Therapie fortgeführt werden.

Eine massive Hämoptoe stellt eine absolute Operationsindikation dar. Weitere tuberkulöse Sekundärkomplikationen wie Aspergillome, chronische aber geringe Hämoptysen oder destroyed lung/-lobe sind unserer Meinung nach relative Indikationen. Die Entscheidung zum chirurgischen Eingriff muss individuell vom klinischen Allgemeinzustand und dem Grad der kardio-pulmonalen Vorschädigung abhängig gemacht werden. Die thoraxchirurgischen Erfahrungen bei derartigen Operationen sind entscheidend für einen komplikationsarmen Verlauf [32]. 


\section{Literatur}

${ }^{1}$ Melvin M, Newman MD. The olden days of surgery for tuberculosis. Ann Thorac Surg 1989; 48: 161 - 162

${ }^{2}$ Maassen W. Pleuro-pulmonary tuberculosis - Current Status of Surgical Therapy. Thorac cardiovasc Surgeon 1980; 28: 225-227

${ }^{3}$ Liebig S. Indikationen zur chirurgischen Behandlung der Lungentuberkulose in der Ära der Kurzzeitchemotherapie. Öff Gesundh.-Wes 1986; 48: 42-48

${ }^{4}$ Teschner M. Aktuelle Indikationen zur Resektion tuberkulöser Lungenerkrankungen - Reminiszenzen der Prächemotherapeutik-Ära? Pneumologie 1998; 52: 80-84

${ }^{5}$ Perelman MI, Strelzov VP. Surgery for pulmonary tuberculosis. World J Surg 1997; 21: 457-467

${ }^{6}$ Lyndtin K. Klinische Ergebnisse als Grundlage für die Indikationsstellung der Chemotherapie und operativen Behandlungen (Dauerresultate). Beiträge Klinik Tbc 1960; 121: 295-298

${ }^{7}$ Moran JF. Surgical treatment of pulmonary tuberculosis. In: Sabiston DC (ed). Textbook of Surgery. 14 ${ }^{\text {th }}$ Ed. IX. 1991

${ }^{8}$ Blaha H. Der intrapleurale, artifizielle Pneumothorax. In: Diethelm L, Olsson O, Strand F, Vieten H, Zuppinger A (Hrsg). Handbuch der medizinischen Radiologie. Berlin: Springer, 1968

${ }^{9}$ Reimers D. Aktive Tuberkulosebehandlung früher - nostalgische Reminiszenzen an eine bewegte Therapieära. Prax Klin Pneumol 1985; 39: $447-456$

${ }^{10}$ Kraan JK, Eerland LD. Indikation zur Resektion wegen Lungentuberkulose und die Resultate dieser Therapie. Beitr Klin Tuberk 1959; 119: 499-515

${ }^{11}$ v. Windheim K. Spezielle Chirurgie für die Praxis. Stuttgart: Georg Thieme Verlag, Band I, Teil 2, 1975: $102-107$

12 Rolle A, Thetter O, Schmölder A, Schweiberer L. Kollapstherapie der Lungentuberkulose - Spätkomplikationen und deren thoraxchirurgische Behandlung. Prax Klin Pneumol 1987; 41: 901

${ }^{13}$ British Thoracic and Tuberculosis Association. Short course chemotherapy in pulmonary tuberculosis. Lancet 1976; 2: $1102-1104$

${ }^{14}$ Lardinois D, Furrer M, Mouton W, Gugger M, Ris HB. Surgical aspects of mycobacteriosis. Evolution during the last 20 years. Schweiz Med Wochenschr 1997; 127: 47: 1961 - 1968

${ }^{15}$ Geldmacher H, Kroeger C, Magnussen H, Kirsten D. Lymphknotentuberkulose in Norddeutschland - eine retrospektive Analyse der Epidemiologie, Diagnosefindung und Therapie bei 60 Patienten im Zeitraum von 1992 - 1999. Zur Publikation eingereicht,

${ }^{16}$ Heckmayr M, Gatzemeier U. Nachweis von Mycobacterium tuberculosis bei atypischer Röntgenmorphologie eines kleinzelligen Bronchialkarzinoms unter Zytostatika-Therapie. Onkologie 1991; 14: $346-351$

${ }^{17}$ Lau SK, Wie WI, Hsu C, Engzell UCG. Efficacy of fine needle aspiration cytology in the diagnosis of tuberculous cervical lymphadenopathy. J Laryngol Otol 1990; 104: 24-27

18 Pomerantz M, Brown J. The surgical management of tuberculosis. Sem Thorac Cardiovasc Surg 1995; 7: 108-111

${ }^{19}$ Liebig S, Holdt H. Indikationen zur Resektionstherapie der Lungentuberkulose. Prax Klein Pneumol 1987; 41: 902 - 903

${ }^{20}$ Klippe HJ. Die Chirurgie in der Therapie pleuropulmonaler Tuberkulosen. Atemw.-Lungenkrkh 1987; 13 (Suppl. 1): 184 185

${ }^{21}$ Iseman MD. Treatment and implications of multidrug-resistent tuberculosis for the $21^{\text {st }}$ century. Chemotherapy 1999; 45 (Suppl 2): $34-40$

${ }^{22}$ Freixinet J. Surgical indications for treatment of pulmonary tuberculosis. World J Surg 1997; 21: 475-479

${ }^{23}$ Riquet M, Souilamas R. Surgery of thoracic and pulmonary tuberculosis and the sequelae of its treatment in adults. Rev Mal Respir 1997; 14 (Suppl 5): 105-120
${ }^{24}$ Hargis JL, Bone RC, Stewart J, Rector N, Hiller FC. Intracavitary amphotericin $\mathrm{B}$ in the treatment of symptomatic pulmonary aspergillomas. Am J Med 1980; 68: 389-394

${ }^{25}$ Klein DL, Gamsu G. Thoracic manifestations of aspergillosis. Am J Roentgenol 1980; 134: 543 - 552

${ }^{26}$ Pichlmaier H, Schildberg FW. Thoraxchirurgie. Berlin, Heidelberg, New York: Springer Verlag, 1987

${ }^{27}$ Chen JC, Chang YL, Luh SP, Lee JM, Lee YC. Surgical treatment for pulmonary aspergilloma: a 28 year experience. Thorac 1997; 52: 810-813

${ }^{28}$ Daly RC, Pairolero PC, Piehler JM, Trastek VF, Bernatz PE. Pulmonary aspergilloma. Results of surgical treatment. J Thorac Cardiovasc Surg 1986; 92: 981 - 988

29 el Oakley R, Petrou M, Goldstraw P. Indications and outcome of surgery for pulmonary aspergilloma. Thorax 1997; 52: 813-815

${ }^{30}$ Stamatis G, Greschuchna D. Surgery for pulmonary aspergilloma and pleural aspergillosis. Thorac Cardiovasc surg 1988; 36 : $356-360$

${ }^{31}$ Schaub R, Klaus D. Tuberkulöse Sekundärinfektion zystischer Lungenveränderungen. Beiträge Klinik Tbc 1959; 120: 415-427

${ }^{32}$ Reed CE, Parker EF, Crawford Jr FA. Surgical resektion for complications of pulmonary tuberculosis. Ann Thorac Surg 1989; 48: $165-167$

Prof. Dr. med. D. Kirsten

Krankenhaus Großhansdorf

Zentrum für Pneumologie und Thoraxchirurgie

Wöhrendamm 80

22927 Großhansdorf 